\title{
Prenatal diagnosis of congenital megalourethra: a rare anomaly
}

\author{
Neha Singh $^{1}$, Vandana Bansal ${ }^{1}$, Kaizad R. Damania ${ }^{2}$
}

\begin{abstract}
${ }^{1}$ Department of Fetal Medicine, Nowrosjee Wadia Maternity Hospital, Mumbai, Maharashtra, India
${ }^{2}$ Department of Obstetrics and Gynecology, Seth GS Medical College and Nowrosjee Wadia Maternity Hospital, Mumbai, Maharashtra, India
\end{abstract}

Received: 30 December 2018

Accepted: 31 January 2018

\author{
*Correspondence: \\ Dr. Vandana Bansal, \\ E-mail: drvandana_bansal@yahoo.co.in
}

Copyright: $@$ the author(s), publisher and licensee Medip Academy. This is an open-access article distributed under the terms of the Creative Commons Attribution Non-Commercial License, which permits unrestricted non-commercial use, distribution, and reproduction in any medium, provided the original work is properly cited.

\begin{abstract}
Congenital megalourethra is a rare urogenital malformation characterised by pathological elongation and dilatation of penile urethra. It is a form of functional obstructive uropathy which is associated with significant urological and sexual dysfunction secondary to hypoplasia or absence of corpora cavernosa and spongiosa. We report a case of megalourethra in one of the dichorionic diamniotic twin diagnosed prenatally in a young primigravida. Initial ultrasound report suggested two different diagnoses i.e. omphalocoele and gastroschisis. Later, diagnosis of congenital megalourethra was made on detailed ultrasound examination by our fetal medicine consultant. Postnatal gross and ultrasonographic examination confirmed the prenatal diagnosis. In the present case report, we have emphasized proper perineal anatomical ultrasound examination which revealed a megalourethra. Although this anomaly is not lethal in isolation, but it has worse prognosis in presence of other associated structural anomalies. This anomaly also raises the controversy regarding disclosure of sex of foetus in countries like India where sex disclosure is prohibited by law. As this condition typically affects male foetuses and significantly affects sexual function, it may be necessary to reveal fetal sex for appropriate counselling and prognostication to prospective parents. Ethical dilemma arises for practising clinicians while dealing with isolated forms of megalourethra whether to offer termination of pregnancy on the grounds of having significant impact on sexual life due to erectile dysfunction. Anorectal malformation is often associated with megalourethra, although suspected in the present case, can be difficult to identify antenatally prior to 20 weeks (the time limit up to which legal termination can be performed in India). These issues have been highlighted in the present report.
\end{abstract}

Keywords: Congenital urogenital anomaly, Hydronephrosis, Lower urinary tract obstruction, Megacystis, Megalourethra, Megapenis, Sexual dysfunction, Urethral cyst, Vesico-amniotic shunting

\section{INTRODUCTION}

Congenital megalourethra is a rare congenital anomaly causing functional obstructive uropathy due to dysgenesis of the penile corpora cavernosa and spongiosa resulting into extensive dilatation of penile urethra. ${ }^{1}$ Benacerraf et al reported first time this condition antenatally in year 1989. Exact embryological etiopathogenesis is not clearly understood yet. Most common accepted theory about genesis of this condition suggest defects in migration, differentiation and development of mesenchymal tissues of phallus. ${ }^{1}$

Dorairajan has classified it into two types based on severity of maldevelopment of penile erectile tissue; scaphoid type and fusiform type. ${ }^{2}$ Scaphoid variant is found to associated with hypoplasia of the corpus spongiosum and bulging of ventral urethra whereas fusiform variant is known to have deficient corpora spongiosa and cavernosa with circumferentially distended 
penile urethra. Scaphoid megalourethra is the milder form. Fusiform variety is more commonly associated with other anomalies and has been associated with worse prognosis.

Prenatal sonographic diagnosis has been reported from 18 weeks onwards and rarely even at 13 weeks of gestation. Classic ultrasound features of congenital megalourethra includes cystic dilatation of penis with or without presence of changes of obstructive uropathy. ${ }^{3}$ More commonly, megalourethra presents as isolated condition with varying degree of functional obstructive uropathy. Almost all cases reported till date have been found to have normal karyotype. ${ }^{1}$ Common associations reported in literature include prune belly syndrome, VACTERL association etc. This condition has also been noted to have several complications like oligohydramnios, renal failure, pulmonary hypoplasia, increased perinatal mortality, urinary and sexual dysfunction., ${ }^{1,3}$ Only few cases have been reported in literature so far.

We report here a case of twin pregnancy affected with this rare condition which closely resembles with prior reported cases published and had anorectal malformation in addition to fusiform megalourethra and changes of obstructive uropathy.

\section{CASE REPORT}

A 22-year-old primigravida with nonconsanguineous marriage who conceived after ovulation induction with dichorionic diamniotic twin gestation, attended our antenatal OPD at 23 weeks of gestation with two outside ultrasound reports suggestive of DCDA twins with anatomically normal first twin and second twin with omphalocoele/gastroschisis with fetal growth restriction and oligohydramnios in second sac. Her antenatal blood investigations were within normal limits including her blood sugar level and thyroid profile. Chorionicity was determined by USG done already outside at 8 weeks gestation.

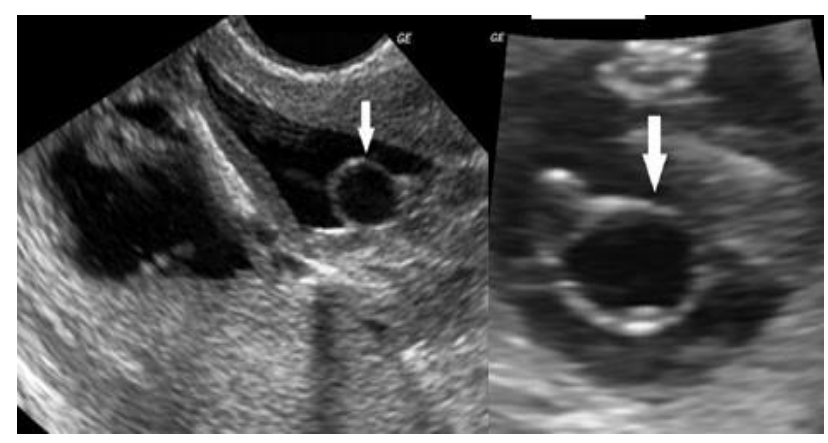

Figure 1: Arrows showing cystic dilatation of distal urethra with thinned walls in perineal region of fetus.

After having registered with us, in view of previous USG findings being inconclusive, detailed fetal anatomical survey was conducted by our fetal medicine consultant.
Sonographic features included symmetric fetal growth restriction, echogenic bowel, single umbilical artery, normal kidneys, normal liquor with dilated urethra (megalourethra) measuring $4.3 \times 1.7 \mathrm{~cm}$ (Figure 1) with normal uterine and umbilical doppler flow velocimetry. Aetiology for FGR in view of above USG findings was probably fetal in origin. Amniocentesis was done for fetal karyotyping to rule out chromosomal abnormalities which came as normal. Paediatric surgery opinion was taken, and prognosis was explained to couple. Presence of megalourethra with echogenic bowel with nonvisualization of fetal anal sphincter (which can be seen on USG as circular hypoechoic area with small echogenic centre), suspicion of VATER complex or anorectal malformation or bowel pathology in fetus was explained to couple with need for postnatal evaluation at birth.

Subsequently follow up scan findings at 34 weeks were suggestive of growth lag by 4 weeks, mild oligohydramnios (largest vertical pocket $2 \mathrm{~cm}$ ), normal doppler, echogenic bowel, distended fetal bladder with left renal pylectasis grade $1(5 \mathrm{~mm})$ as shown in Figure 2 below.

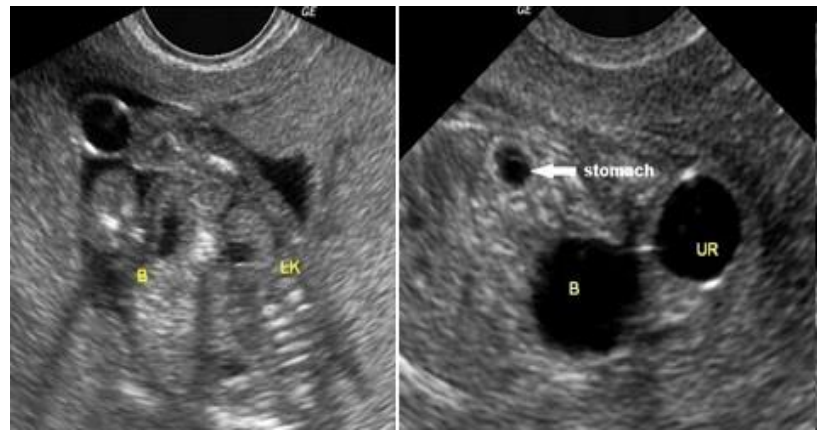

B: Bladder; LK: Left kidney; UR: Urethra

Figure 2: Megalourethra with left renal pylectasis.

At 37 weeks gestation, further progression of disease condition was noted in form of bilateral renal pelviectasis grade 2 with oligohydramnios (LVP $1.2 \mathrm{~cm}$ ) and growth restriction with effective fetal weight of $1.6 \mathrm{~kg}$ (along third centile) with megalourethra measuring $4.2 \times 1.9 \mathrm{~cm}$ (shown in Figure 3).

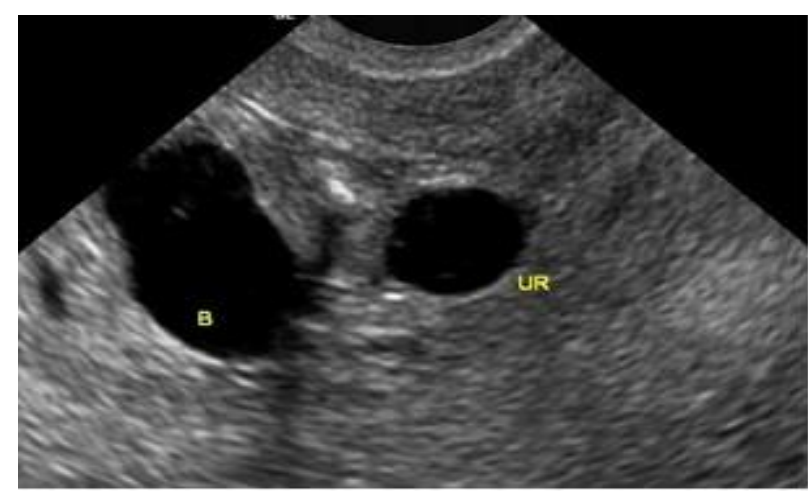

Figure 3: Megalourethra (UR) with distended bladder (B). 
On the other hand, first twin showed appropriate interval growth and normal doppler flows in all scans. She was delivered by emergency caesarean section at 37.1 weeks in view of both twins in breech presentation. First twin was a female child who weighed $2.39 \mathrm{~kg}$ and cried immediately after birth with APGAR score of 9/10 with normal gross features. She had an uneventful postnatal course in NICU and normal USG skull, abdomen and KUB findings.

Second twin was a male child weighing $1.57 \mathrm{~kg}$ who did not initiate spontaneous respiration at birth. Baby was resuscitated and transferred to NICU immediately in view of respiratory distress. On gross examination, baby had single umbilical artery, imperforate anus and large elongated penis as shown in Figure 4 below.

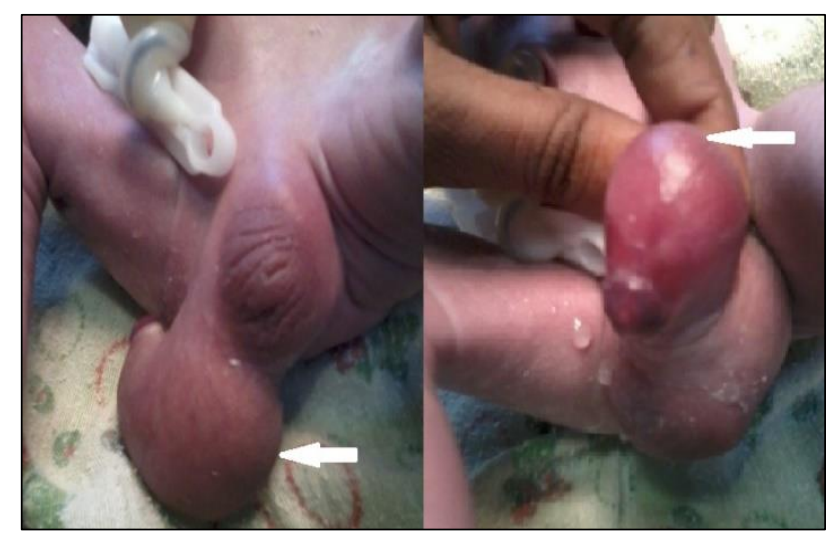

Figure 4: Gross examination of second twin showing elongated and distended penis (shown with arrows) and bulging of ventral surface consistent with diagnosis of megalourethra.

Postnatal USG findings were consistent with antenatal USG findings along with an imperforate anus.

In view of gross congenital structural malformations and guarded prognosis even after surgical correction and increased expenses in treatment, couple refused to undergo further treatment of affected twin and took discharge against medical advice on day 5 of delivery. Baby died on day 7 of his birth and 2 days after discharge from hospital.

\section{DISCUSSION}

Antenatal diagnosis of megalourethra can be made by its hallmark sonographic features like cystic structure in perineal region, hydronephrosis and distended bladder with or without hydroureter. ${ }^{3}$ In most of the cases, amniotic fluid remains normal in amount. Differential diagnosis may include two congenital abnormalities of anterior urethra i.e. anterior urethral valve and diverticulum. But, these two conditions are less likely to cause balloon dilatation of penis. ${ }^{4}$
It is always recommended that careful examination of anterior abdominal wall and perineal region must be done to avoid error in identifying difference between loop of umbilical cord or gastroschisis or omphalocoele and thin walled urethral cyst; as happened in the present case where diagnosis was initially given as anterior abdominal wall defect instead of megalourethra.

Amsalem et al published the largest case series of ten cases of congenital megalourethra reported so far in literature. All ten cases were identified to have megalourethra at a median gestational age of 19 weeks (range, 13-24 weeks). Diagnosis of megalourethra was confirmed postnatally or at autopsy in all cases. All cases presented with a distended bladder and megalourethra with normal karyotype. They concluded that diagnosis of megalourethra should be considered in all male fetuses presenting antenatally with megacystis on ultrasound and detailed fetal anatomical survey on USG should be done to look for a distended and / or elongated phallic structure and any other associated anomalies. ${ }^{1}$ Although very rare, megalourethra has been described in twin pregnancies by Johannes et al. ${ }^{5}$

Management options available antenatally include vesico-amniotic shunting and fetoscopic decompression of distal urethral obstruction. Migliorelli et al published a case of congenital megalourethra managed successfully with use of fetoscopy to treat severe lower urinary tract obstruction in a male fetus who presented at 21 weeks with massive dilatation of penile urethra and changes of obstructive uropathy. Fetoscopy revealed absence of urethral meatus. Contact diode laser perforation of tip of penis was performed. They concluded that fetoscopic decompression of a distal urethral obstruction can achieve neonatal survival in these cases. ${ }^{6}$

Postnatal diagnosis can be made with gross examination and imaging modalities like retrograde urethrogram and cystourethroscopy. Various treatment options include vesicostomy, ureterostomy, pyelotomy, reduction urethroplasty and penile prosthesis in adults having fusiform variety. Although, role of above mentioned treatment modalities is still being evaluated in trials.

Fetal and neonatal prognosis depends upon presence of oligohydramnios, degree of renal dysfunction, lung hypoplasia and presence of other associated anomalies. In isolated cases, quality of life in terms of urinary and sexual dysfunction depends on ability to correct the urologic dysfunction.

\section{Clinical significance}

The main aim of early diagnosis in antenatal period is to provide parents with an accurate diagnosis and prognosis. Thus, it allows them to make an informed decision regarding continuation or termination of pregnancy. 
Presence of megalourethra on USG should raise the suspicion of other associated anomalies like anorectal malformations, VATER complex, prune belly syndrome which may worsen prognosis. Most such cases will have normal karyotype. Isolated form of megalourethra detected on ultrasound, needs a detailed counselling of the parents regarding the sexual/erectile/urological dysfunction of the child at birth and later in life.

It raises the controversy regarding disclosure of sex of fetus in countries like India where sex disclosure is prohibited by law. As this condition typically affects male foetuses and significantly affects sexual function, it may be necessary to reveal fetal sex for appropriate counselling and prognostication.

This condition also gives rise to ethical dilemma for clinicians in offering termination in view of impaired sexual function later in adulthood when present in isolated form.

\section{CONCLUSION}

Megalourethra results in significant urinary and sexual dysfunction (e.g. erectile/ejaculatory dysfunction) unlike other causes of lower urinary tract obstruction. Such cases may need several urosurgical interventions in postnatal period and subsequently later in life. Therefore, making accurate and earlier diagnosis is of extreme importance as it affects management and prognostication significantly. In India where sex determination is legally not allowed, mentioning the disorder would disclose the sex. Counselling of prospective parents would become extremely difficult without disclosure of the sex.
Funding: No funding sources Conflict of interest: None declared

Ethical approval: Not required

\section{REFERENCES}

1. Amsalem H, Fitzgerald B, Keating S, Ryan G. Congenital megalourethra: prenatal diagnosis and postnatal/autopsy findings in 10 cases. Ultrasound Obstet Gynecol. 2011;37:678-83.

2. Dorairajan T. Defects of spongy tissue and congenital diverticula of the penile urethra. Aust N Z J Surg. 1962;32:209-14.

3. Lam YH, Tang MHY. Sonographic diagnosis of congenital megalourethra at 13 weeks of gestation. Ultrasound Obstet Gynecol. 2000;16:585-6.

4. Jones EA, Freedman AL, Ehrlich RM. Megalourethra and urethral diverticula. Urol Clin North Am. 2002;29:341-8.

5. Van Der Merwea JL, Rademana K, Geertsa L. Congenital megalourethra in monochorionic twins: a case report. Lippincott Williams and Wilkins. Clin Dysmorphol. 2013;22(1).

6. Migliorelli F, Martínez JM, Gómez O, Bennasar M. Successful fetoscopic surgery to release a complete obstruction of the urethral meatus in a case of congenital megalourethra. Fetal Diagn Ther. 2015;38(1):77-80.

Cite this article as: Singh N, Bansal V, Damania KR. Prenatal diagnosis of congenital megalourethra: a rare anomaly. Int J Reprod Contracept Obstet Gynecol 2018;7:1251-4. 